\title{
A DELAY CALIBRATION SYSTEM FOR TWO-WAY SATELLITE TIME AND FREQUENCY TRANSFER
}

\author{
F. G. ASCARRUNZ, S. R. JEFFERTS AND T. E. PARKER \\ NIST, 325 Broadway, Boulder CO $80303^{*}$
}

\section{Introduction}

We describe a system used to calibrate delays in two-way satellite time and frequency transfer (TWSTFT) earth stations. The calibration system is designed to measure the transmit $T X(t)$ and receive $R X(t)$ delays through the earth station in real time allowing for the possibility of real-time correction of TWSTFT data. The delay calibration device is stabilized to a temperature of $45 \pm 3{ }^{\circ} \mathrm{C}$. The measured peak-to-peak delay noise of the calibration device in loop-back operation with no common mode cancellation of delay errors is $800 \mathrm{ps}$. The time stability $\sigma_{\mathrm{x}}(\tau)$ is on the order of 100 ps out to an averaging time of 3 days.

\section{Two satellite time and frequency transfer}

The main components of the earth station $[1,2]$ at the National Institute of Standards and Technology (NIST) that is normally used in TWSTFT are shown in Figure 1. The timing information of the local clock is contained in a

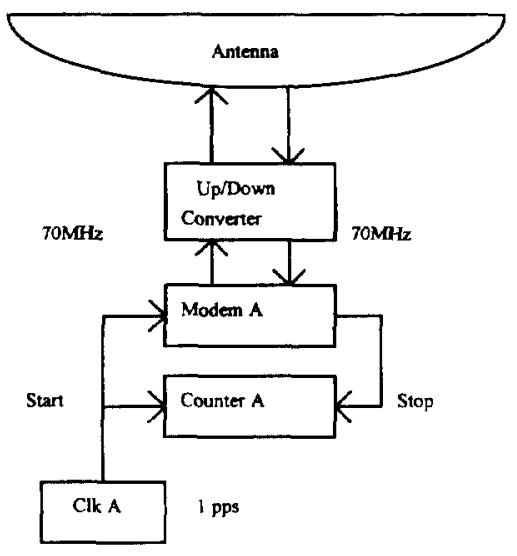

Figure 1. TWSTFT Earth Station.

one pulse per second ( $1 \mathrm{pps}$ ) signal. The local 1 pps signal starts a local counter and is encoded on a $70 \mathrm{MHz}$ carrier by a modem. The $70 \mathrm{MHz}$ signal is routed through a long
$(50 \mathrm{~m})$ cable to the antenna site. At the antenna site the 70 $\mathrm{MHz}$ signal is up-converted to the transmit frequency in the range of 14 to $14.5 \mathrm{GHz}$. The $\mathrm{Ku}$-band signal is transmitted via a communications satellite to the remote site. The satellite transponder down-converts the 14 to $14.5 \mathrm{GHz}$ signals to the range of 11.7 to $12.2 \mathrm{GHz}$. The $\mathrm{Ku}$-band signals in the frequency range of 11.7 to 12.2 $\mathrm{GHz}$ from the remote earth station are received by the antenna and down-converted to $70 \mathrm{MHz}$ by the down converter at the antenna site. The $70 \mathrm{MHz}$ signal containing the remote timing information is relayed to the modem through a $50 \mathrm{~m}$ cable. The modem decodes the remote lpps signal that stops the local counter. The difference of the time intervals measured by the local and remote counters yields a measure of the time difference between the local and remote clocks. The difference in time between the clocks at the local site (designated by the A subscript) and the clock at the remote site (designated by the $B$ subscript) is given by equation (1). clk $\mathrm{k}_{\mathrm{i}}$ is the time at clock $i, T C_{i}(t)$ is the time between the transmitted one pulse per second (1pps) and the received lpps measured by

$$
c l k_{A}-c l k_{B}=\frac{1}{2}\left\{\begin{array}{l}
T C_{B}(t)-T C_{A}(t)+T x_{B}(t)-R x_{B}(t)- \\
T x_{A}(t)+R x_{A}(t)+P_{B, A}(t)-P_{A \cdot B}(t)
\end{array}\right\}+S_{A B}
$$

the time interval counter at station $I$ and $T x_{i}(t)$ is the transmit delay from the clock through the modem, cables and earth station at location $i$. $\operatorname{Rx}_{i}(t)$ is the receive delay from the earth station, the modem and cables to the counter at location i. $P_{i, j}(t)$ is the time delay for the signal to go from location $i$ through the satellite transponder to location j. $S_{i j}$ is the Sagnac effect, the path non-reciprocity due to the rotation of the earth for locations $i$ and $j$.

The unknown terms in equation (1) are the difference in path delays and the receive and transmit delays through the earth stations at the two sites. The receive and transmit delay through the earth station could be due to group delay changes, the effective shortening or lengthening of the transmission paths. This type of delay can be measured using the delay calibration device $[3,4]$. In addition to the changes in the effective group delay through the receive and transmit paths, there are also apparent delay changes due to biases in the decorrelation of the spread spectrum signals in the modem $[5,6]$. These 
apparent delay changes cannot be measured using the delay calibration device. The group delay through the transmit path of the earth station will be designated as $\operatorname{TXg}(t)$ and the group delay through the receive path of the earth station will be designated as $\mathrm{RXg}(\mathrm{t})$.

\section{Delay calibration unit}

The delay calibration unit consists of an upconverter and a down-converter. The up-converter takes a $70 \mathrm{MHz}$ signal and up-converts the signal to a selected frequency in the frequency band of $11.7 \mathrm{GHz}$ to $12.2 \mathrm{GHz}$. The frequency resolution of the up-converter is $100 \mathrm{~Hz}$. The down-converter takes a signal with a selected frequency in the $14 \mathrm{GHz}$ to $14.5 \mathrm{GHz}$ frequency band and down-converts it to $70 \mathrm{MHz}$. The frequency resolution of the down-converter is also $100 \mathrm{~Hz}$. A block diagram of the delay calibration unit is shown in figure 2 . The up and down-converters were designed to minimize group delays, time delay instabilities and time delay sensitivity to environmental conditions. The wide-band design of the delay calibration unit minimized the group delay through

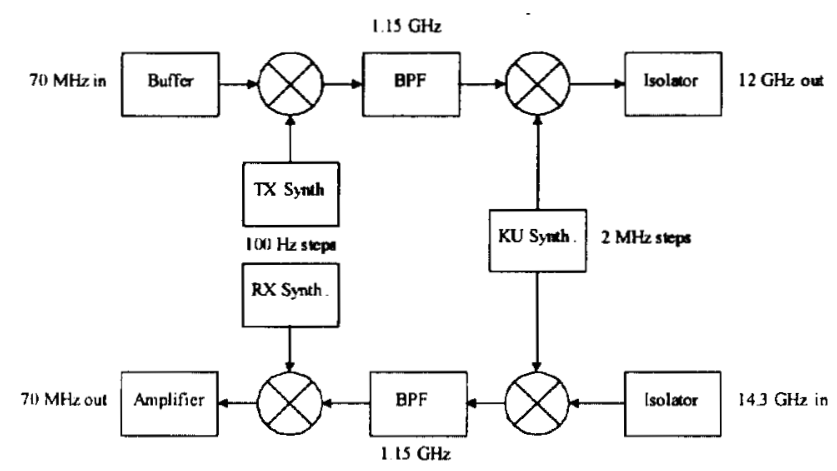

Figure 2. Delay calibration unit.

the device. The delay through a calibration unit receiver plus the delay through the calibration unit transmitter was measured to be $351 \pm 1$ ns. Temperature insensitive substrates were used for the bandpass filters centered at $1.15 \mathrm{GHz}$. The temperature coefficient of delay through the calibration unit was measured to be approximately 10 $\mathrm{ps} /{ }^{\circ} \mathrm{C}$ for ambient temperatures of 25 to $65{ }^{\circ} \mathrm{C}$. The temperature coefficient of delay for the calibration unit is more than an order of magnitude better than the temperature coefficient of delay of the earth stations at NIST [2]. The delay calibration unit contains a microprocessor that continually monitors phase-locked loop voltages and the temperature of the unit. The user communicates with the calibration unit via RS232 to set the frequency of the up and down converter and to monitor the temperature and status of the device. The enclosure of the calibration unit is temperature stabilized to $45 \pm 3{ }^{\circ} \mathrm{C}$.
Since the temperature coefficient of delay through the device is $10 \mathrm{ps} /{ }^{\circ} \mathrm{C}$, the peak-to-peak changes in delay due to temperature sensitivity do not exceed 60ps.

The delay calibration unit is installed on an earth station to allow monitoring and real-time measurement of the earth station transmit $\mathrm{TXg}(\mathrm{t})$ and receive $\mathrm{RXg}(\mathrm{t})$ delays. The block diagram of the modified TWSTFT equipment setup is shown in figure 3 . The delay calibration system components include: (1) a delay

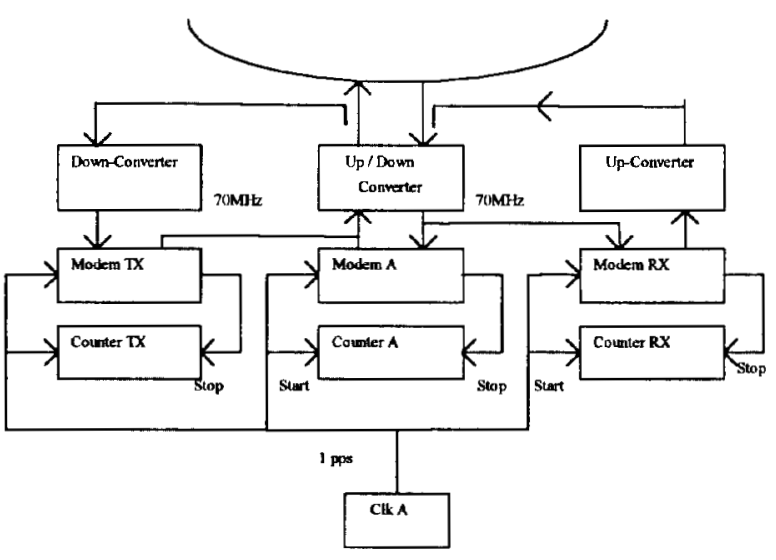

Figure 3. Installed delay calibration system.

calibration unit with its up and down converters, (2) two NIST modems, (3) a counter to measure the transmit path delay (TX counter), (4) a counter to measure the receive path delay (RX counter), (5) the wave guide couplers, (6) the transmit and receive path $70 \mathrm{MHz}$ cables and (7) the temperature control system. The modems are located in the lab along with the counters and clocks. The $70 \mathrm{MHz}$ cables are approximately $50 \mathrm{~m}$ long and connect the modems to the delay calibration unit.

The transmit path delay through the earth station is measured by sampling the transmitted signal at the waveguide feed of the antenna with the use of a directional coupler. The sampled signal is down-converted to 70 $\mathrm{MHz}$ and the timing information, lpps, is decoded in a modem. This lpps signal stops the TX counter that was started by the transmitted $1 \mathrm{pps}$. The reading on the counter measures the propagation delay through the transmit section of the earth station and the calibration unit. The receive path delay through the earth station is measured by having a lpps signal start the RX counter. The $1 \mathrm{pps}$ is encoded on a $70 \mathrm{MHz}$ carrier by a modem. The $70 \mathrm{MHz}$ signal is up-converted in the calibration unit to the receiver frequency of the earth station and coupled into the earth station antenna feed. The injected signal is down-converted by the earth station to $70 \mathrm{MHz}$ and a 
modem is used to decode the lpps signal that stops the RX counter. The reading on the $\mathrm{RX}$ counter is the delay through the earth station receiver plus the delay through the calibration unit.

\section{Stability of the Delay Calibration System}

The time-delay stability of the delay calibration system was measured with the system installed on a very small aperture terminal (VSAT) earth station at NIST. During these measurements the VSAT earth station was not in operation. The stability of the delay calibration system was being tested in what would be its operational configuration. The delay calibration system up-converter was connected through a short cable to the delay calibration down-converter. This loop-back configuration allows the measurement of the sum of the delays through the modems, cables, up-converter and down-converter of the delay calibration system. This sum of delays gives an upper bound on the maximum time delay instability of the delay calibration system. Measurements, of the sum of the delays through the delay calibration system in loop-back mode were taken every hour for a period of two weeks. Each hourly point is an average of three hundred one per second measurements. The outside temperature was also recorded during this period. Figures (4) and (5) display

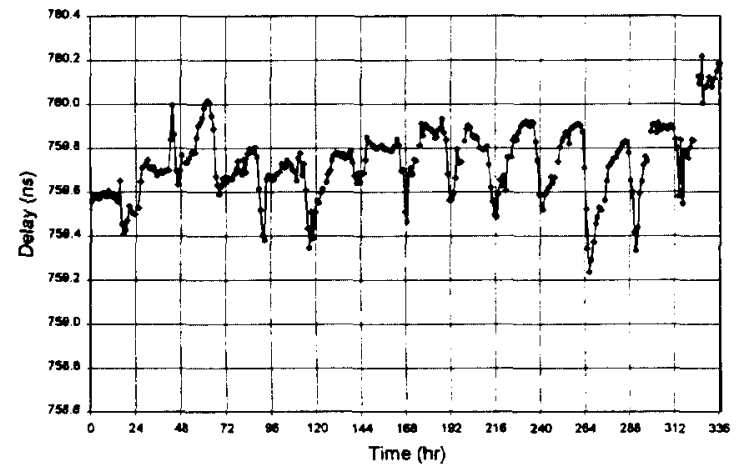

Figure 4. Plot of time delay through calibration system for a two-week period.

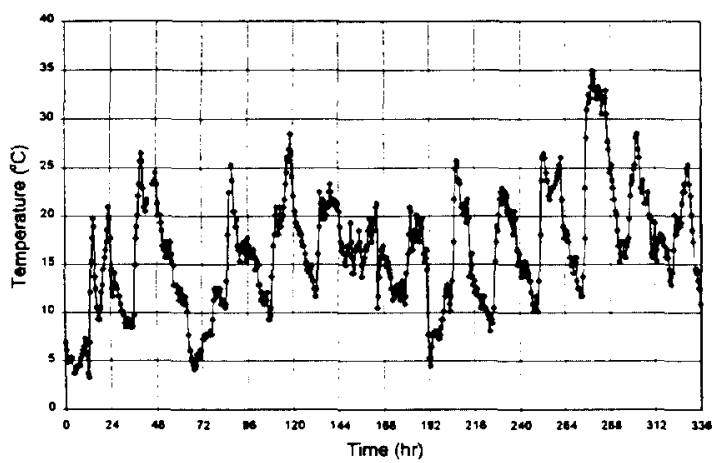

Figure 5. Temperature data for the 2 week period. plots of the delay and temperature during this two week period. There is a delay dependence on temperature (about $20 \mathrm{ps} /{ }^{\circ} \mathrm{C}$ ) that is not caused by the delay calibration unit, since the delay calibration unit is temperature controlled at $45 \pm 3^{\circ} \mathrm{C}$. Figure (6) shows the time delay stability of the

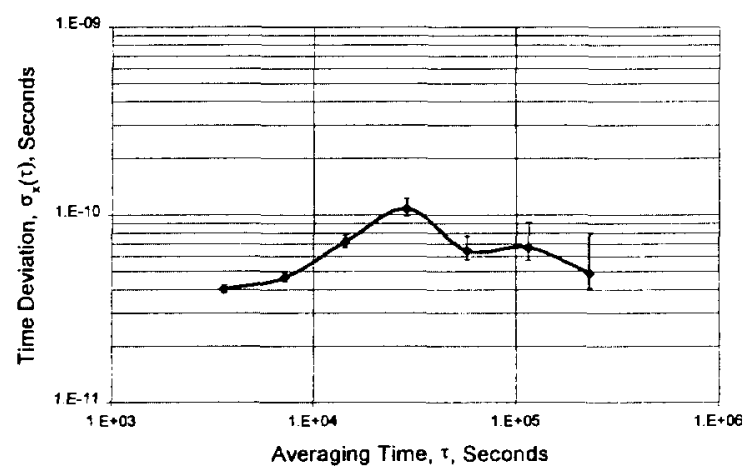

Figure 6. Time stability plot of calibration unit data.

calibration system during this two-week period. The system time delay stability is about 100 ps for averaging times out to three days.

\section{Conclusion}

The delay calibration system can be used to measure the transmit and receive path delay instabilities in earth stations used for two-way satellite time and frequency transfer. The upper bound on the delay instability of the delay measurement system $\left(\sigma_{x}(\tau)\right)$ is 100 ps out to averaging times of three days. The system can be used as a diagnostic tool to determine the components of the earth station giving rise to large delay instabilities. The system can also be used to implement real-time corrections for delay instabilities in the earth stations. The delay calibration system cannot be used to measure apparent delay instabilities that are caused by biases in the demodulation of the lpps signals.

\section{References}

1. C. Hackman, S. R. Jefferts and Thomas E. Parker, "Common clock two-way satellite time transfer experiments," in Proc. 1995 IEEE Frequency Control Symposium, 1995, pp. 275-281.

2. F. G. Ascarrunz, S. R. Jefferts and Thomas E. Parker, "Earth Station Errors in Two-way Time and Frequency Transfer," in IEEE Transactions on Instrumentation and Measurement, Vol 46, No. 2, 1997. 
3. G. de Jong, "Delay Stability of the TWSTFT Earth Station at VSL," in Proc. $29^{\text {th }}$ Annual Precise Time and Time Interval Meeting, 1997.

4. D. Kirchner, H. Ressler and R. Robnik, "An Automated signal delay monitoring system for a twoway satellite time transfer terminal," in Proc. European Frequency and Time Forum, pp. 75-79, 1995.

5. R. C. Dixon, Spread Spectrum Systems with Commercial Applications, Wiley 1994.

6. D. A. Howe, "Time Tracking Error in DirectSequence Spread Spectrum Networks Due to Coherence Among Signals", IEEE Trans. on Communications, Vol. 38, No. 12, 1990. 